\title{
The Mellin transform of The square of Riemann's ZETA-FunCtion
}

\author{
AleKsandar Ivić
}

\begin{abstract}
Let $\mathcal{Z}_{1}(s)=\int_{1}^{\infty}\left|\zeta\left(\frac{1}{2}+i x\right)\right|^{2} x^{-s} \mathrm{~d} x(\sigma=\Re e s>1)$. A result concerning analytic continuation of $Z_{1}(s)$ to $\mathbb{C}$ is proved, and also a result relating the order of $\mathcal{Z}_{1}(\sigma+i t)\left(\frac{1}{2} \leq \sigma \leq 1, t \geq t_{0}\right)$ to the order of $\mathcal{Z}_{1}\left(\frac{1}{2}+i t\right)$.
\end{abstract}

\section{Introduction}

Let $\mathcal{Z}_{k}(s)$, the (modified) Mellin transform of $\left|\zeta\left(\frac{1}{2}+i x\right)\right|^{2 k}$, denote the analytic continuation of the function defined initially by

$$
\mathcal{Z}_{k}(s):=\int_{1}^{\infty}\left|\zeta\left(\frac{1}{2}+i x\right)\right|^{2 k} x^{-s} \mathrm{~d} x \quad(k \in \mathbb{N}, \sigma=\Re \mathrm{e} s>c(k)(>1)) .
$$

This function, when $k=2$, was introduced by Y. Motohashi [15] (see also [16]), and its properties were further studied in [10] and [11]. The latter work also contains some results on the function $\mathcal{Z}_{1}(s)$, which is the principal object of the study in this paper. It was shown that $\mathcal{Z}_{1}(s)$ is regular for $\sigma>-3 / 4$, except for a double pole at $s=1$. The principal part of the Laurent expansion of $\mathcal{Z}_{1}(s)$ at $s=1$ is

$$
\frac{1}{(s-1)^{2}}+\frac{2 \gamma-\log (2 \pi)}{s-1}
$$

where $\gamma=-\Gamma^{\prime}(1)=0.577215 \ldots$ is Euler's constant. M. Jutila [13] continued the study of $\mathcal{Z}_{1}(s)$ and proved that $\mathcal{Z}_{1}(s)$ continues meromorphically to $\mathbb{C}$, having only a double pole at $s=1$ and at most double poles for $s=-1,-2, \ldots$

The object of this note is to prove some new results on $\mathcal{Z}_{1}(s)$. First we make more precise Jutila's result on the analytic continuation of $\mathcal{Z}_{1}(s)$. We have the following

THEOREM 1. The function $\mathcal{Z}_{1}(s)$ continues meromorphically to $\mathbb{C}$, having only a double pole at $s=1$, and at most simple poles at $s=-1,-3, \ldots$ The principal part of its Laurent expansion at $s=1$ is given by (1.2).

Our second aim is to prove an order result for $\mathcal{Z}_{1}(s)$. This is a result M. Jutila mentioned in [13], but the term that I initially claimed, namely $t^{1-2 \sigma+\varepsilon}$ is too optimistic. It appears that what can be proved is contained in

THEOREM 2. We have, for $\frac{1}{2} \leq \sigma \leq 1, t \geq t_{0}>0$,

$$
\mathcal{Z}_{1}(\sigma+i t) \ll_{\varepsilon} t^{\frac{1}{2}-\sigma+\varepsilon} \max _{t-t^{\varepsilon} \leq v \leq t+t^{\varepsilon}}\left|\mathcal{Z}_{1}\left(\frac{1}{2}+i v\right)\right|+\left(t^{\frac{9-16 \sigma}{7}}+t^{-1}\right) \log t .
$$

2001 Mathematics Subject Classification. 11M06 
Corollary (see M. Jutila [13]). For $\frac{1}{2} \leq \sigma \leq 1, t \geq t_{0}$ we have

$$
\mathcal{Z}_{1}(\sigma+i t) \ll_{\varepsilon} t^{\frac{5}{6}-\sigma+\varepsilon}
$$

The bound $\mathcal{Z}_{1}\left(\frac{1}{2}+i t\right) \ll_{\varepsilon} t^{1 / 3+\varepsilon}$ was mentioned in [11], and its proof was elaborated by M. Jutila in [13]. If one inserts this bound in (1.3), then (1.4) follows immediately. We note that here and later $\varepsilon$ denotes arbitrarily small constants, not necessarily the same ones at each occurrence. It can be also proved that, for any given $\varepsilon>0$,

$$
\int_{1}^{T}\left|\mathcal{Z}_{k}(\sigma+i t)\right|^{2} \mathrm{~d} t \gg_{\varepsilon} T^{2-2 \sigma-\varepsilon} \quad\left(k=1,2 ; \frac{1}{2}<\sigma<1\right) .
$$

The bound (1.5) for $k=2$ appeared in my paper [10], and the proof of the bound when $k=1$ is on similar lines, so that the details will not be given here. It is plausible that

$$
\max _{t-t^{\varepsilon} \leq v \leq t+t^{\varepsilon}}\left|\mathcal{Z}_{1}\left(\frac{1}{2}+i v\right)\right| \gg 1
$$

holds, but at present I am unable to prove (1.6).

\section{Proof of Theorem 1}

Let

$$
L_{k}(s):=\int_{0}^{\infty}\left|\zeta\left(\frac{1}{2}+i x\right)\right|^{2 k} \mathrm{e}^{-s x} \mathrm{~d} x \quad(\sigma=\Re \mathrm{e} s>0, k \in \mathbb{N})
$$

be the Laplace transform of $\left|\zeta\left(\frac{1}{2}+i x\right)\right|^{2 k}$. We are interested in using the expression for $L_{1}(s)$ when $s=1 / T, T \rightarrow \infty$. Such a formula has been known for a long time and is due to H. Kober [14] (see also a proof in [17, Chapter 9]). The functions $L_{k}(s)$ when $k=1,2$ were studied by F.V. Atkinson [1], [2]. The function $L_{2}(s)$ was considered by the author [8], [9], and the approach to mean value of Dirichlet series by Laplace transforms by $\mathrm{M}$. Jutila [12]. Kober's result on $L_{1}(s)$ is that, for any integer $N \geq 0$,

$$
L_{1}(\sigma)=\frac{\gamma-\log (2 \pi \sigma)}{2 \sin \frac{\sigma}{2}}+\sum_{n=0}^{N} d_{n} \sigma^{n}+O_{N}\left(\sigma^{N+1}\right) \quad(\sigma \rightarrow 0+) \text {, }
$$

where $d_{n}$ are suitable constants. Note that for $\sigma=1 / T, T \rightarrow \infty$ we have

$$
\begin{aligned}
& \frac{\gamma-\log \left(\frac{2 \pi}{T}\right)}{2 \sin \left(\frac{1}{2 T}\right)}=\frac{\log \left(\frac{T}{2 \pi}\right)+\gamma}{\frac{1}{T}-\frac{1}{24 T^{3}}+\frac{1}{16 \cdot 5 ! T^{5}}-\cdots} \\
& =\left(\log \left(\frac{T}{2 \pi}\right)+\gamma\right) \sum_{n=0}^{\infty} c_{n} T^{1-2 n}
\end{aligned}
$$


with the coefficients $c_{n}$ that may be explicitly evaluated. Therefore for any integer $N \geq 0$

$$
L_{1}\left(\frac{1}{T}\right)=\left(\log \left(\frac{T}{2 \pi}\right)+\gamma\right) \sum_{n=0}^{N} a_{n} T^{1-2 n}+\sum_{n=0}^{N} b_{n} T^{-2 n}+O_{N}\left(T^{-1-2 N} \log T\right)
$$

with the coefficients $a_{n}, b_{n}$ that may be explicitly evaluated. In particular, we have

$$
a_{0}=1, \quad b_{0}=\pi
$$

It is clear from Kober's formula (2.2) that $a_{0}=1$. To see that $b_{0}=\pi$ one can use the work of Hafner-Ivić [4] (this was mentioned by Conrey et al. [3]), which will be stated now in detail, as it will be needed also for the proof of Theorem 2 . Let, as usual, for $T \geq 0$,

$$
E(T)=\int_{0}^{T}\left|\zeta\left(\frac{1}{2}+i t\right)\right|^{2} \mathrm{~d} t-T\left(\log \left(\frac{T}{2 \pi}\right)+2 \gamma-1\right) \quad(E(0)=0)
$$

denote the error term in the mean square formula for $\left|\zeta\left(\frac{1}{2}+i t\right)\right|$. Let further

$$
G(T):=\int_{0}^{T} E(t) \mathrm{d} t-\pi T, \quad G_{1}(T):=\int_{0}^{T} G(t) \mathrm{d} t .
$$

Then Hafner-Ivić [4] proved (see also [6])

$$
G(T)=S_{1}(T ; N)-S_{2}(T ; N)+O\left(T^{1 / 4}\right)
$$

with

$$
\begin{gathered}
S_{1}(T ; N)=2^{-3 / 2} \sum_{n \leq N}(-1)^{n} d(n) n^{-1 / 2}\left(\operatorname{arsinh} \sqrt{\frac{\pi n}{2 T}}\right)^{-2}\left(\frac{T}{2 \pi n}+\frac{1}{4}\right)^{-1 / 4} \sin (f(T, n)), \\
S_{2}(T ; N)=\sum_{n \leq N^{\prime}} d(n) n^{-1 / 2}\left(\log \frac{T}{2 \pi n}\right)^{-2} \sin (g(T, n)), \\
f(T, n)=2 T \operatorname{arsinh} \sqrt{\frac{\pi n}{2 T}}+\sqrt{2 \pi n T+\pi^{2} n^{2}}-\frac{\pi}{4} \\
g(T, n)=T \log \left(\frac{T}{2 \pi n}\right)-T+\frac{\pi}{4}, \operatorname{arsinh} x=\log \left(x+\sqrt{x^{2}+1}\right), \\
A T<N<A^{\prime} T\left(0<A<A^{\prime} \text { constants }\right), N^{\prime}=\frac{T}{2 \pi}+\frac{N}{2}-\sqrt{\frac{N^{2}}{4}+\frac{N T}{2 \pi}} .
\end{gathered}
$$

We use Taylor's formula (see [7, Lemma 3.2]) to simplify (2.6). Then we obtain

$$
G(T)=2^{-1 / 4} \pi^{-3 / 4} T^{3 / 4} \sum_{n=1}^{\infty}(-1)^{n} d(n) n^{-5 / 4} \sin \left(\sqrt{8 \pi n T}-\frac{\pi}{4}\right)+O\left(T^{2 / 3} \log T\right),
$$


so that

$$
G(T)=O\left(T^{3 / 4}\right), \quad G(T)=\Omega_{ \pm}\left(T^{3 / 4}\right) .
$$

We use (2.6) with $T=t, N=T, T \leq t \leq 2 T$ and apply the first derivative test ([5, Lemma 2.1]) to deduce that

$$
\int_{T}^{2 T} G(t) \mathrm{d} t \ll T^{5 / 4},
$$

since the term $O(1)$ in $[5$, eq. (15.29)] in Atkinson's formula is in fact $O(1 / T)$. Hence

$$
G_{1}(T)=\int_{0}^{T} G(t) \mathrm{d} t \ll T^{5 / 4}
$$

To prove that $b_{0}=\pi$ (cf. (2.4)) we note that, using the definition of $E(T)$ and applying integration by parts,

$$
\begin{aligned}
& L_{1}\left(\frac{1}{T}\right)=\int_{0}^{\infty} \mathrm{e}^{-t / T}\left(\log \left(\frac{t}{2 \pi}\right)+2 \gamma+E^{\prime}(t)\right) \mathrm{d} t \\
& =T \int_{0}^{\infty} \mathrm{e}^{-x}\left(\log x+\log \frac{T}{2 \pi}+2 \gamma\right) \mathrm{d} x+\frac{1}{T} \int_{0}^{\infty} E(t) \mathrm{e}^{-t / T} \mathrm{~d} t \\
& =T\left(\log \left(\frac{T}{2 \pi}\right)+\gamma\right)+\frac{1}{T^{2}} \int_{0}^{\infty} \int_{0}^{t} E(u) \mathrm{d} u \cdot \mathrm{e}^{-t / T} \mathrm{~d} t \\
& =T\left(\log \left(\frac{T}{2 \pi}\right)+\gamma\right)+\frac{1}{T^{2}} \int_{0}^{\infty}\left(\pi t+O\left(t^{3 / 4}\right)\right) \mathrm{e}^{-t / T} \mathrm{~d} t \\
& =T\left(\log \left(\frac{T}{2 \pi}\right)+\gamma\right)+\pi+O\left(T^{-1 / 4}\right),
\end{aligned}
$$

where we also used the $O$-bound of $(2.7)$ and

$$
\int_{0}^{\infty} \mathrm{e}^{-x} \log x \mathrm{~d} x=\Gamma^{\prime}(1)=-\gamma
$$

A comparison of (2.3) and (2.9) proves that $b_{0}=\pi$, as asserted by (2.4).

We return now to the proof of Theorem 1. Let

$$
\bar{L}_{k}(s):=\int_{1}^{\infty}\left|\zeta\left(\frac{1}{2}+i y\right)\right|^{2 k} \mathrm{e}^{-y s} \mathrm{~d} y \quad(k \in \mathbb{N}, \sigma=\Re \mathrm{e} s>0) .
$$

Then we have by absolute convergence, taking $\sigma$ sufficiently large and making the change of variable $x y=t$,

$$
\begin{aligned}
& \int_{0}^{\infty} \bar{L}_{k}(x) x^{s-1} \mathrm{~d} x=\int_{0}^{\infty}\left(\int_{1}^{\infty}\left|\zeta\left(\frac{1}{2}+i y\right)\right|^{2 k} \mathrm{e}^{-y x} \mathrm{~d} y\right) x^{s-1} \mathrm{~d} x \\
& =\int_{1}^{\infty}\left|\zeta\left(\frac{1}{2}+i y\right)\right|^{2 k}\left(\int_{0}^{\infty} x^{s-1} \mathrm{e}^{-x y} \mathrm{~d} x\right) \mathrm{d} y \\
& =\int_{1}^{\infty}\left|\zeta\left(\frac{1}{2}+i y\right)\right|^{2 k} y^{-s} \mathrm{~d} y \int_{0}^{\infty} \mathrm{e}^{-t} t^{s-1} \mathrm{~d} t=\mathcal{Z}_{k}(s) \Gamma(s) .
\end{aligned}
$$


Further we have

$$
\begin{aligned}
& \int_{0}^{\infty} \bar{L}_{1}(x) x^{s-1} \mathrm{~d} x=\int_{0}^{1} \bar{L}_{1}(x) x^{s-1} \mathrm{~d} x+\int_{1}^{\infty} \bar{L}_{1}(x) x^{s-1} \mathrm{~d} x \\
& =\int_{1}^{\infty} \bar{L}_{1}(1 / x) x^{-1-s} \mathrm{~d} x+A(s) \quad(\sigma>1),
\end{aligned}
$$

where

$$
A(s):=\int_{1}^{\infty} \bar{L}_{1}(x) x^{s-1} \mathrm{~d} x
$$

is an entire function. Since

$$
\bar{L}_{1}(1 / x)=L_{1}(1 / x)-\int_{0}^{1}\left|\zeta\left(\frac{1}{2}+i y\right)\right|^{2} \mathrm{e}^{-y / x} \mathrm{~d} y \quad(x \geq 1),
$$

it follows from (2.11) with $k=1$ by analytic continuation that, for $\sigma>1$,

$$
\begin{aligned}
\mathcal{Z}_{1}(s) \Gamma(s) & =\int_{1}^{\infty} L_{1}(1 / x) x^{-1-s} \mathrm{~d} x-\int_{1}^{\infty}\left(\int_{0}^{1}\left|\zeta\left(\frac{1}{2}+i y\right)\right|^{2} \mathrm{e}^{-y / x} \mathrm{~d} y\right) x^{-1-s} \mathrm{~d} x+A(s) \\
& =I_{1}(s)-I_{2}(s)+A(s),
\end{aligned}
$$

say. Clearly for any integer $M \geq 1$

$$
\begin{aligned}
I_{2}(s) & =\int_{1}^{\infty} \int_{0}^{1}\left|\zeta\left(\frac{1}{2}+i y\right)\right|^{2}\left(\sum_{m=0}^{M} \frac{(-1)^{m}}{m !}\left(\frac{y}{x}\right)^{m}+O_{M}\left(x^{-M-1}\right)\right) \mathrm{d} y x^{-1-s} \mathrm{~d} x \\
& =\sum_{m=0}^{M} \frac{(-1)^{m}}{m !} h_{m} \cdot \frac{1}{m+s}+H_{M}(s),
\end{aligned}
$$

say, where $H_{M}(s)$ is a regular function of $s$ for $\sigma>-M-1$, and

$$
h_{m}:=\int_{0}^{1}\left|\zeta\left(\frac{1}{2}+i y\right)\right|^{2} y^{m} \mathrm{~d} y
$$

is a constant. Inserting (2.3) in $I_{1}(s)$ in (2.12) we have, for $\sigma>1$,

$$
\begin{aligned}
I_{1}(s) & =\int_{1}^{\infty}\left(\log \frac{x}{2 \pi}+\gamma\right) \sum_{n=0}^{N} a_{n} x^{-2 n-s} \mathrm{~d} x+\int_{1}^{\infty} \sum_{n=0}^{N} b_{n} x^{-1-2 n-s} \mathrm{~d} x+K_{N}(s) \\
& =\sum_{n=0}^{N} a_{n}\left(\frac{1}{(2 n+s-1)^{2}}+\frac{\gamma-\log 2 \pi}{2 n+s-1}\right)+K_{N}(s),
\end{aligned}
$$

say, where $K_{N}(s)$ is regular for $\sigma>-2 N$. Taking $M=2 N$ it follows from $(2.12)-(2.14)$ that

$$
\begin{aligned}
\mathcal{Z}_{1}(s) \Gamma(s) & =\sum_{n=0}^{N} a_{n}\left(\frac{1}{(2 n+s-1)^{2}}+\frac{\gamma-\log 2 \pi}{2 n+s-1}\right) \\
& +\sum_{m=0}^{2 N} \frac{(-1)^{m}}{m !} h_{m} \cdot \frac{1}{m+s}+R_{N}(s),
\end{aligned}
$$


say, where $R_{N}(s)$ is a regular function of $s$ for $\sigma>-2 N$. This holds initially for $\sigma>1$, but by analytic continuation it holds for $\sigma>-2 N$. Since $N$ is arbitrary and $\Gamma(s)$ has no zeros, it follows that $(2.15)$ provides meromorphic continuation of $\mathcal{Z}_{1}(s)$ to $\mathbb{C}$. Taking into account that $\Gamma(s)$ has simple poles at $s=-m(m=0,1,2, \ldots)$ with residues $\frac{(-1)^{m}}{m !}$, and that near $s=1$ we have the Taylor expansion

$$
\frac{1}{\Gamma(s)}=1+\gamma(s-1)+\sum_{n=2}^{\infty} \frac{f_{n}}{n !}(s-1)^{n}
$$

with $f_{n}=\left.(1 / \Gamma(s))^{(n)}\right|_{s=1}$, it follows that

$$
\begin{aligned}
\mathcal{Z}_{1}(s) & =\frac{1}{(s-1)^{2}}+\frac{2 \gamma-\log (2 \pi)}{s-1} \\
& +\frac{1}{\Gamma(s)}\left\{\sum_{n=1}^{N} a_{n}\left(\frac{1}{(2 n+s-1)^{2}}+\frac{\gamma-\log 2 \pi}{2 n+s-1}\right)\right\} \\
& +\frac{1}{\Gamma(s)}\left(\sum_{m=0}^{2 N} \frac{(-1)^{m}}{m !} h_{m} \cdot \frac{1}{m+s}\right)+U_{N}(s),
\end{aligned}
$$

say, where $U_{N}(s)$ is a regular function of $s$ for $\sigma>-2 N$. Moreover, the function $1 /(\Gamma(s)(2 n+s-1))$ is regular for $n \in \mathbb{N}$ and $s \in \mathbb{C}$. Therefore (2.16) provides analytic continuation to $\mathbb{C}$, showing that besides $s=1$ the only poles of $\mathcal{Z}_{1}(s)$ can be simple poles at $s=1-2 n$ for $n \in \mathbb{N}$, as asserted by Theorem 1 . With more care the residues at these poles could be explicitly evaluated.

\section{Proof Theorem 2}

We suppose that $X \gg t$ and start from

$$
\mathcal{Z}_{1}(s)=\left(\int_{1}^{X}+\int_{X}^{\infty}\right)\left|\zeta\left(\frac{1}{2}+i x\right)\right|^{2} x^{-s} \mathrm{~d} x \quad(\sigma=\Re \mathrm{e} s>1)
$$

We use $\left|\zeta\left(\frac{1}{2}+i x\right)\right|^{2}=\log (x / 2 \pi)+2 \gamma+(E(x)-\pi)^{\prime}$ and integrate by parts to obtain

$$
\begin{aligned}
\mathcal{Z}_{1}(s) & =\int_{1}^{t^{1-\varepsilon}}\left|\zeta\left(\frac{1}{2}+i x\right)\right|^{2} x^{-s} \mathrm{~d} x+\int_{t^{1-\varepsilon}}^{X}\left|\zeta\left(\frac{1}{2}+i x\right)\right|^{2} x^{-s} \mathrm{~d} x \\
& +\frac{X^{1-s}}{s-1}\left(\frac{1}{s-1}+\log X+2 \gamma-\log 2 \pi\right) \\
& -(E(X)-\pi) X^{-s}+s \int_{X}^{\infty}(E(x)-\pi) x^{-s-1} \mathrm{~d} x \\
& =I_{1}+I_{2}+O\left(X^{-\sigma}+t^{-1} X^{1-\sigma} \log X\right)+I_{3},
\end{aligned}
$$


provided $E(X)=0$. But as proved in [6], every interval $[T, T+C \sqrt{T}]$ with sufficiently large $C>0$ contains a zero of $E(t)$, hence we can assume that $E(X)=0$ is fulfilled with suitable $X$. Since $E(x)$ is $\asymp x^{1 / 4}$ in mean square (see e.g., [5] or [7]), it follows by analytic continuation that (3.1) is valid for $\sigma>\frac{1}{4}, t \geq t_{0}>0, t \leq X \leq t^{A}(A \geq 1)$. By repeated integration by parts it is found that $I_{1} \ll t^{-1}$. The integral $I_{2}$ is split in $O(\log T)$ subintegrals of the form

$$
J_{K}=\int_{K / 2}^{5 K / 2} \varphi(x)\left|\zeta\left(\frac{1}{2}+i x\right)\right|^{2} x^{-s} \mathrm{~d} x
$$

where $\varphi(x) \in C^{\infty}$ is a nonnegative, smooth function supported in $[K / 2,5 K / 2]$ that is equal to unity in $[K, 2 K]$. To bound $J_{K}$ one can start from (1.1) with $k=1$ and use the Mellin inversion formula (see [10] for a detailed discussion concerning the analogous situation with the fourth moment of $\left.\left|\zeta\left(\frac{1}{2}+i t\right)\right|\right)$, which yields

$$
\left|\zeta\left(\frac{1}{2}+i x\right)\right|^{2}=\frac{1}{2 \pi i} \int_{(1+\varepsilon)} \mathcal{Z}_{1}(s) x^{s-1} \mathrm{~d} s \quad(x>1) .
$$

Here we replace the line of integration by the contour $\mathcal{L}$, consisting of the same straight line from which the segment $[2+\varepsilon-i, 1+\varepsilon+i]$ is removed and replaced by a circular arc of unit radius, lying to the left of the line, which passes over the pole $s=1$ of the integrand. By the residue theorem we have

$$
\left|\zeta\left(\frac{1}{2}+i x\right)\right|^{2}=\frac{1}{2 \pi i} \int_{\mathcal{L}} \mathcal{Z}_{1}(s) x^{s-1} \mathrm{~d} s+\log \left(\frac{x}{2 \pi}\right)+2 \gamma \quad(x>1) .
$$

This gives

$$
J_{K}=\frac{1}{2 \pi i} \int_{\mathcal{L}} \mathcal{Z}_{1}(w) \int_{K / 2}^{5 K / 2} \varphi(x) x^{w-s-1} \mathrm{~d} x \mathrm{~d} w+O\left(t^{-1}\right)
$$

Repeated integration by parts in the $x$-integral shows that only the portion $\mid \Im m w-$ $\Im \mathrm{m} s \mid \leq t^{\varepsilon}$ gives a non-negligible contribution. Hence replacing $\mathcal{L}$ by the line $\Re$ e $w=\frac{1}{2}$ we obtain

$$
J_{K} \ll_{\varepsilon} K^{\frac{1}{2}-\sigma} t^{\varepsilon} \max _{t-t^{\varepsilon} \leq v \leq t+t^{\varepsilon}}\left|\mathcal{Z}_{1}\left(\frac{1}{2}+i v\right)\right|+t^{-1}
$$

To estimate $I_{3}$ in (3.1) we integrate by parts and use (2.7) and (2.8). We obtain

$$
\begin{aligned}
& \int_{X}^{\infty}(E(x)-\pi) x^{-s-1} \mathrm{~d} x=-G(X) X^{-s-1}+(s+1) \int_{X}^{\infty} G(x) x^{-s-2} \mathrm{~d} x \\
& =O\left(X^{-1 / 4-\sigma}\right)-(s+1) G_{1}(X) X^{-s-2}+(s+1)(s+2) \int_{X}^{\infty} G_{1}(x) x^{-s-3} \mathrm{~d} x \\
& \ll X^{-1 / 4-\sigma}+t^{2} X^{-3 / 4-\sigma} .
\end{aligned}
$$


Thus it follows from (3.1) and (3.2) that we have, for $\frac{1}{2} \leq \sigma \leq 1, t \geq t_{0}$,

$$
\begin{aligned}
\mathcal{Z}_{1}(\sigma+i t) & \ll_{\varepsilon} t^{\frac{1}{2}-\sigma+\varepsilon} \max _{t-t^{\varepsilon} \leq v \leq t+t^{\varepsilon}}\left|\mathcal{Z}_{1}\left(\frac{1}{2}+i v\right)\right|+\left(X^{1-\sigma} t^{-1}+t^{-1}\right) \log t \\
& +X^{-\sigma}+t X^{-1 / 4-\sigma}+t^{3} X^{-3 / 4-\sigma}
\end{aligned}
$$

In (3.3) we choose $X$ to satisfy

$$
X^{1-\sigma} t^{-1}=t^{3} X^{-3 / 4-\sigma}, \quad \text { i.e., } \quad X=t^{16 / 7} .
$$

Then $t X^{-1 / 4-\sigma}+X^{-\sigma} \ll t^{3} X^{-3 / 4-\sigma}$, and (1.3) follows from (3.3).

In conclusion, one may ask what should be the true order of magnitude of $\mathcal{Z}_{1}(\sigma+i t)$. This is a difficult question, but it seems reasonable to expect that

$$
\mathcal{Z}_{1}(\sigma+i t) \ll_{\varepsilon} t^{\frac{1}{2}-\sigma+2 \mu\left(\frac{1}{2}\right)+\varepsilon} \quad\left(\frac{1}{2} \leq \sigma \leq 1, t \geq t_{0}>0\right)
$$

holds, where

$$
\mu(\sigma):=\limsup _{t \rightarrow \infty} \frac{\log |\zeta(\sigma+i t)|}{\log t}
$$

is the Lindelöf exponent of $\zeta(s)$. One could try to obtain (3.4) from (1.3) by refining the estimation of $\mathcal{Z}_{1}(s)$ in [11]. This procedure leads to an exponential sum with the divisor function which is estimated as $\ll_{\varepsilon} t^{2 \mu\left(\frac{1}{2}\right)+\varepsilon}$ (this, of course, is non-trivial and needs elaboration).

\section{REFERENCES}

[1] F.V. Atkinson, The mean value of the zeta-function on the critical line, Quart. J. Math. Oxford 10(1939), 122-128.

[2] F.V. Atkinson, The mean value of the zeta-function on the critical line, Proc. London Math. Soc. 47(1941), 174-200.

[3] J.B. Conrey, D.W. Farmer, J.P. Keating, M.O. Rubinstein and N.C. Snaith, Integral moments of $L$-functions, 71pp, ArXiv.math.NT/0206018.

[4] J.L. Hafner and A. Ivić, On the mean square of the Riemann zeta-function on the critical line, J. Number Theory 32(1989), 151-191.

[5] A. Ivić, The Riemann zeta-function, John Wiley and Sons, New York, 1985.

[6] A. Ivić, Large values of certain number-theoretic error terms, Acta Arith. 56(1990), 135-159.

[7] A. Ivić, Mean values of the Riemann zeta-function, LN's 82, Tata Institute of Fundamental Research, Bombay, 1991 (distr. by Springer Verlag, Berlin etc.).

[8] A. Ivić, On the fourth moment of the Riemann zeta-function, Publs. Inst. Math. (Belgrade) 57(71) (1995), 101-110.

[9] A. Ivić, The Laplace transform of the fourth moment of the zeta-function, Univ. Beograd. Publ. Elektrotehn. Fak. Ser. Mat. 11(2000), 41-48. 
[10] A. Ivić, On some conjectures and results for the Riemann zeta-function, Acta. Arith. 99(2001), 115-145.

[11] A. Ivić, M. Jutila and Y. Motohashi, The Mellin transform of powers of the Riemann zeta-function, Acta Arith. 95(2000), 305-342.

[12] M. Jutila, Mean values of Dirichlet series via Laplace transforms, in "Analytic Number Theory" (ed. Y. Motohashi), London Math. Soc. LNS 247, Cambridge University Press, Cambridge, 1997, 169-207.

[13] M. Jutila, The Mellin transform of the square of Riemann's zeta-function, Periodica Math. Hung. 42(2001), 179-190.

[14] H. Kober, Eine Mittelwertformel der Riemannschen Zetafunktion, Compositio Math. 3(1936), 174-189.

[15] Y. Motohashi, A relation between the Riemann zeta-function and the hyperbolic Laplacian, Annali Scuola Norm. Sup. Pisa, Cl. Sci. IV ser. 22(1995), 299-313.

[16] Y. Motohashi, Spectral theory of the Riemann zeta-function, Cambridge University Press, 1997.

[17] E.C. Titchmarsh, The Theory of the Riemann Zeta-Function (2nd ed.), Clarendon Press, Oxford, 1986.

Aleksandar Ivić

Katedra Matematike RGF-A

Universitet U Beogradu

ĐUŠINA 7, 11000 BEOGRAD

Serbia and Montenegro

E-MAIL: aivic@matf.bg.ac.yu,

ivic@rgf.bg.ac.yu 